(c) Elsevier/INRA

Article original

\title{
Paramètres génétiques des caractères de croissance, de gavage et de foie gras dans le croisement de deux souches d'oies (Anser anser) sélectionnées
}

\author{
R Rouvier ${ }^{1}$, B Poujardieu ${ }^{1}$ D Rousselot-Pailley ${ }^{2}$ \\ P Larrue $^{2}$, D Esteve ${ }^{3}$ \\ ${ }^{1}$ Institut national de la recherche agronomique, centre de recherches de Toulouse, \\ station d'amélioration génétique des animaux, BP 27, F 31326 Castanet-Tolosan; \\ ${ }^{2}$ Institut national de la recherche agronomique, station expérimentale de l'oie, \\ Artiguères, BP 68, 40002 Mont-de-Marsan; \\ ${ }^{3}$ Centre technique de la conserve des produits animaux, station expérimentale de l'oie, \\ Artiguères, BP 68, 40002 Mont-de-Marsan, France
}

(Reçu le 7 mars 1990; accepté le 16 décembre 1991)

Résumé - Les performances de croissance, de gavage, d'abattage, et de fonte lipidique à la stérilisation de foies gras de 611 oisons (318 mâles et 293 femelles) de 8 types génétiques sont analysées. Ils sont obtenus en 2 générations successives comparées simultanément : croisement factoriel de 2 souches d'oies sélectionnées, INRA Landaise 01 (L01) et Sepalm (SE), et croisement de jars de la souche INRA Landaise 00 (LO0) avec des oies de ces 2 souches et leurs 2 métisses réciproques. Les 8 valeurs génotypiques moyennes des oisons sont estimées suivant un modèle linéaire qui prend en compte les effets fixés du type génétique, du sexe et leur interaction, du lot de gavage, et l'âge à la mise en gavage comme covariable. Six paramètres génétiques du croisement sont obtenus, suivant le modèle de Dickerson (1969). Les effets des gènes liés au sexe sont introduits dans le modèle.

Dès l'âge de 8 semaines, les mâles sont plus lourds que les femelles; ils gardent cet avantage jusqu'à la fin du gavage. Les foies des mâles, bien que plus lourds, sont moins fondants que ceux des femelles. La souche SE, de format supérieur à la souche L01, croît plus vite et produit un foie plus lourd de qualité technologique plus faible.

Les décompositions génétiques des caractères pondéraux corporels sont semblables : effets génétiques directs et maternels favorables de la souche Sepalm et en général significatifs. Les effets grand-maternels ont tendance à être en opposition, ils ne sont significatifs que pour le poids en début de gavage. Les hétérosis directs sont négatifs, significatifs pour les poids à l'âge de 8 semaines et en début de gavage, les hétérosis maternels sont favorables et en général significatifs. La décomposition du poids du paletot s'en distingue par l'absence 
d'antagonisme entre effets maternel et grand-maternel. Les décompositions des gains de poids sont différentes : les paramètres du croisement y sont statistiquement nuls sauf pour l'hétérosis direct sur le gain de poids 8 semaines-prégavage, les hétérosis direct et maternel pour le gain en cours de gavage, qui sont positifs.

Pour le poids du foie gras, la souche Sepalm se caractérise par des effets génétiques directs des gènes autosomaux $\left(g_{s e}^{I}=204 \pm 70 \mathrm{~g}\right)$ et des gènes liés au sexe $\left(g_{s e}^{I S}=72 \pm 70 \mathrm{~g}\right)$ qui sont favorables et en opposition avec ses effets grand-maternels $\left(g_{s e}^{N}=-55 \pm 22 \mathrm{~g}\right)$. Ses effets génétiques directs sur le taux de fonte lipidique sont également forts $\left(g_{s e}^{I}=8 \% \pm 1,5 \%\right)$.

Des hétérosis direct et maternel négatifs diminuent la fonte lipidique. Il est conclu que la sélection de souches et leur croisement peuvent permettre d'améliorer les caractères d'élevage ainsi que le poids et la qualité technologique des foies gras d'oies des 2 sexes.

oie / croisement / croissance / foie gras / fonte lipidique

Summary - Genetic effects on growth, force feeding and fatty liver traits in crosses of 2 selected geese (Anser anser) strains. Growth, force feeding, slaughter, fat release from the fatty liver traits for 611 goslings (318 males and 293 females) from 8 genotypes are analysed. Two successive generations are studied: factorial crossbreeding of 2 selected strains, INRA Landaise 01 (LO1) and Sepalm (SE), and crossbreeding of ganders from INRA Landaise 00 (LOO) with females of these 2 strains and 2 reciprocal crossbreds. The 8 genotypic mean values of goslings are estimated according to a linear model taking into account fixed effects of genotype, sex and their interaction, force feeding batch, and the age at the beginning of force feeding as a covariate. Six crossbreeding genetic parameters are estimated, following the Dickerson (1969) model. Direct effects of sex linked genes are introduced. From 8 weeks of age up to the end of force feeding males are heavier than females. Fatty livers from males are heavier and with less fat release after sterilisation than those of females. The Sepalm strain is bigger than the L01 strain, has a higher growth rate and produces heavier fatty liver with lower technological quality.

Decomposition into genetic effects is similar for all body weight traits : positive direct and maternal effects of the Sica Sepalm strain, antagonisms with grand-maternal effects, small contribution of sex linked genes; direct heterosis effects are negative and significant only for body weights at 8 weeks of age and at the beginning of force feeding; maternal heterosis effects are positive, not significant only for the body weight at the beginning of pre-force feeding. For paletot weight there is no antagonism between maternal and grandmaternal effects. Decomposition of body weight gains is different : genetic parameters are not significant, except direct heterosis for weight gain between 8 weeks of age and pre-force feeding, and direct and maternal heterosis for weight gain during force feeding, which are positive. For fatty liver weight, the Sepalm strain has quite high direct genetic effects for autosomal genes ( $\left(\mathrm{g}_{\mathrm{SE}}^{\mathrm{I}}=204 \pm 25 \mathrm{~g}\right.$ ) and for sex linked genes $\left(\mathrm{g}_{\mathrm{SE}}^{\mathrm{Is}}=72 \pm 70 \mathrm{~g}\right.$ ), which are antagonistic to grand-maternal effects ( $\mathrm{g}_{\mathrm{SE}}^{\mathrm{N}}=-55 \pm 22 \mathrm{~g}$ ). Direct genetic effects for fat release are also high ( $\mathrm{g} \mathrm{SE}=8 \% \pm 1.5 \%)$, but maternal and grand-maternal genetic effects for this trait are not significant. The complementarity between genetic effects explains the improvement in fatty liver production and the lowering in the sexual dimorphism in fatty liver weight when crossing Sepalm gander with L01 female goose. There is no heterosis effect for fatty liver weight. But crossing of these 2 strains lowers fat release for a given fatty liver weight, by direct and mainly maternal heterosis effects. It is concluded that selection and crossbreeding of strains can improve rearing performances and also fatty liver weight and technological quality in both sexes.

goose / crossbreeding / growth / fatty liver / fat release 


\section{INTRODUCTION}

La définition de l'utilisation optimale de souches sélectionnées suppose l'estimation des paramètres génétiques de leur croisement. Si cette estimation a été réalisée chez les mammifêres (par exemple Brun et Rouvier, 1984, 1988 chez le lapin; Menissier et al, 1988, chez les bovins de races à viande), ce n'était pas encore le cas, à notre connaissance, pour les oies grasses. Deux souches d'oies Landaises, INRA L00 et L01, ainsi qu'une souche d'oie Grise du Sud-Ouest de la France, d'origines génétiques différentes, sont sélectionnés en souche pure, depuis plusieurs générations, respectivement à la station expérimentale de l'oie Artiguères (Rouvier et al, $1982 \mathrm{a}, \mathrm{b}$ ) et par la Sica Sepalm à Souprosse (souche SE) en collaboration avec l'INRA. L'objectif des recherches est d'améliorer l'aptitude génétique des souches pour la prolificité des femelles, le poids du foie gras après un gavage court, la qualité technologique des foies, la qualité des paletots des oisons gavés.

Une expérimentation a donc été réalisée dans le but d'estimer les paramètres génétiques du croisement des souches L01 et Sepalm (SE), pour les caractères de croissance, de gavage, de poids et qualité technologique des foies gras des oisons élevés et gavés. Les jars L00 ont été utilisés comme jars testeurs communs dans des croisements 2 et 3 souches. Il a paru utile d'en donner et discuter ici les résultats.

\section{MATÉRIEL ET MÉTHODE}

\section{Matériel biologique et protocole expérimental}

La constitution des 2 souches d'oies Landaises L01 et L00 de la station expérimentale de l'oie Artiguères, les objectifs et leur méthode de sélection ont été décrits précédemment (Rouvier et al, 1982b). Les 2 souches sont à leur cinquième génération de sélection au moment de cette expérimentation. Les souches L00 et L01, de plus petit format que la souche Sepalm, étaient sélectionnées expérimentalement, la première pour accroître le poids du foie gras, la deuxième pour accroître la production d'oisons des femelles. Une souche d'oie Grise du Sud Ouest a été constituée en 1979 par la Sica Sepalm, organisme interprofessionnel, à partir d'animaux de plusieurs élevages soumis au contrôle de performances en ferme. L'objectif de sélection est l'accroissement du poids, de la qualité du foie gras, et de la production d'oisons. Les reproducteurs Sepalm utilisés dans cette expérimentation sont issus de la deuxième génération de sélection.

L'expérimentation s'est déroulée en 1988 dans les installations de la Station expérimentale de l'oie à Artiguères. Les oisons mâles et femelles mesurés descendent en deuxième ponte en cycle naturel de reproducteurs conduits selon le dispositif schématisé au tableau I. Ce dispositif est la juxtaposition au même moment d'un croisement factoriel des souches pures Landaises 01 (L01) et Sepalm (SE) et d'un croisement d'un testeur mâle commun de la souche Landaise 00 (L00) avec des femelles de 4 génotypes produits du premier type de croisement.

Les reproducteurs sont conduits à l'extérieur en parquets de groupe sur caillebotis. Chaque parquet dispose d'un bassin de copulation.

Les oisons expérimentaux éclosent en 5 lots entre le 22 mars et le 17 mai 1988. De la naissance à l'âge de 5 semaines, les oisons, placés sous éleveuse au sol, consomment 
Tableau I. Dispositif expérimental du plan de croisement.

\begin{tabular}{|c|c|c|c|c|c|c|}
\hline Génotype père & $L 01 \quad L 01$ & $S E \quad S E$ & $L 00$ & $L 00$ & $L 00$ & $L 00$ \\
\hline Génotype mère & $L 01 \quad S E$ & $L 01 \quad S E$ & $L 01$ & $S E \times L 01$ & $L 01 \times S E$ & $S E$ \\
\hline Type de parquets & \multicolumn{2}{|c|}{$\begin{array}{c}\text { Par type de croisement } \\
3 \text { parquets } \\
\text { ( } 3 \text { mâles } \times 9 \text { femelles) }\end{array}$} & \multicolumn{4}{|c|}{$\begin{array}{c}\text { Par type de croisement } \\
3 \text { parquets } \\
(3 \text { mâles } \times 9 \text { femelles }) \\
1 \text { parquet } \\
\text { (16 mâles } \times 48 \text { femelles })\end{array}$} \\
\hline
\end{tabular}

à volonté un aliment complet dosant $2850 \mathrm{Kcal} \mathrm{EM} / \mathrm{kg}$ et de 16 à $18 \%$ de MAT ( $1 \%$ de lysine et $0,85 \%$ d'acides aminés soufrés). Entre les âges de 6 et 8 semaines, ils consomment à volonté un aliment dosant $2850 \mathrm{kcal} \mathrm{EM} / \mathrm{kg}$ et de 14 à $15 \%$ de MAT $(0,7 \%$ de lysine et $0,8 \%$ d'acides aminés soufrés). À l'âge de 8 semaines révolues, ils sont placés sur caillebotis à une densité de l'ordre de 3 oisons au $\mathrm{m}^{2}$. Ils consomment alors jusqu'au début du gavage un aliment dosant $2600 \mathrm{kcal} \mathrm{EM} / \mathrm{kg}$ et $14 \%$ de MAT $\left(0,6 \%\right.$ de lysine et d'acides aminés soufrés) rationné à $160 \mathrm{~g} \cdot \mathrm{j}^{-1}$ par oison jusqu'au début du prégavage puis à volonté. Le prégavage dure $14 \mathrm{j}$.

Les oisons ont été gavés en 4 séries qui ont débuté le 16 août, le 5 septembre, le 27 septembre et le 7 novembre. Les âges moyens à la mise en gavage étaient dans l'ordre des séries de $130 \mathrm{j}, 139 \mathrm{j}, 149 \mathrm{j}$ et $182 \mathrm{j}$. Quatre gaveurs, toujours les mêmes, interviennent à chaque série. Chaque gaveur reçoit de façon équilibrée des mâles, des femelles et des oisons des différents génotypes. Le gavage est réalisé à l'aide de maïs mi-cuit distribué en 3 repas quotidiens avec repasse. La durée du gavage n'excède pas $15 \mathrm{j}$. Des animaux ont été abattus dès $9 \mathrm{j}$ après la mise en gavage, car le gaveur est laissé libre d'apprécier le moment où la stéatose hépatique atteind son optimum. Les animaux sont saignés (après électronarcose), plumés, mis en chambre froide à $+4^{\circ} \mathrm{C}$. L'éviscération, la pesée du foie, la prise d'un échantillon de $60 \mathrm{~g}$ mis en boîte sertie pour la stérilisation, la découpe et la pesée du paletot ont lieu $24 \mathrm{~h}$ après l'abattage.

\section{Caractères mesurés et analysés}

Les poids des oisons à la naissance, à l'âge de 8 semaines, à la mise en prégavage, au début et à la fin de gavage, le poids ressuyé (saigné et plumé), les poids du foie et du paletot sont mesurés individuellement. Les gains moyens journaliers entre l'âge de 8 semaines et le début du prégavage, pendant le prégavage et pendant le gavage sont calculés. La qualité technologique est mesurée par le poids de l'exsudat lipidique d'un morceau de $60 \mathrm{~g}$ de foie découpé dans le haut du lobe droit après stérilisation en autoclave à valeur stérilisatrice de 1 . Elle est exprimée en pourcentage d'un morceau de foie de $100 \mathrm{~g}$ (taux de fonte pourcent) et en écart à la régression, tous génotypes et sexes confondus, du poids de l'exsudat lipidique sur le poids du foie («fonte écart»). Le taux de fonte est le critère vrai de qualité technologique des foies en conserverie. Il a été apprécié indirectement par une mesure au qualimètre; cet appareil, mis au point par Salladare (1978) mesure l'impédance bioélectrique du 
tissu hépatique. La corrélation avec la fonte est de l'ordre de 0,8 (Bartel, 1984). La variable considérée est la moyenne de 3 mesures prises dans le haut du lobe droit.

\section{Méthode d'analyse}

Les paramètres du croisement (Dickerson, 1969) ont été estimés à partir des performances moyennes des 8 génotypes. La décomposition de ces performances moyennes des oisons mâles et femelles en fonction des paramètres du croisement est présentée dans le tableau II. Six paramètres sont estimables à partir du dispositif expérimental utilisé : les différences entre races pour les effets directs $\left(g_{S E-01}^{I}\right)$, maternels $\left(g_{S E-01}^{M}\right)$ et grand-maternels $\left(g_{S E-01}^{N}\right)$ des gènes autosomaux et pour les effets liés au sexe $\left(g_{S E-01}^{I_{s}}\right)$, les effets d'hétérosis direct $\left(h_{01 \times S E}^{I}\right)$ et maternel $\left(h_{01 \times S E}^{M}\right)$ du croisement entre les lignées $S E$ et 01 . La décomposition des performances moyennes des 8 génotypes chez les oisons mâles et femelles ne diffère que par les coefficients de $g^{I s}$.

Les estimées des paramètres du croisement sont calculées à l'aide des fonctions estimables décrites au tableau III et construites sur des estimées des effets génotypiques obtenus du modèle :

$$
Y_{i j k l}=m+t_{i}+p_{j}+s_{k}+(t \cdot s)_{i k}+b\left(x_{i j k l}-\bar{x}\right)+e_{i j k l}
$$

où $y_{i j k l}$ est la performance du $1^{\mathrm{e}}$ oison de type génétique $i(i=1-8)$, de sexe $k$ $(k=1,2)$ gavé à l'âge de $x_{i j k l} \mathrm{j}$ dans le $\mathrm{j}^{\mathrm{e}}$ lot de gavage $(j=1-6)$. Cet âge est considéré comme covariable, $b$ étant le coefficient de régression. Le lot de gavage est défini par la combinaison série de gavage $\times$ gaveur. L'âge de mise en gavage n'est introduit dans le modèle que pour les caractères mesurés depuis le début du gavage. Pour estimer les effets génétiques qui nécessitent de distinguer les performances des mâles de celles des femelles, nous avons remplacé dans le modèle ci-dessus, les effets du type génétique, du sexe et de leur interaction, par l'effet combiné du sexe et du type génétique (16 modalités). La procédure GLM de SAS est utilisée pour obtenir les estimées des effets fixés par la méthode des moindres carrés et en même temps celles des fonctions estimables construites à partir des effets types génétiques ainsi que leurs écarts types qui servent pour le test de leur signification (SAS Institute, 1985), sur IBM 3083.

L'introduction dans le modèle de l'âge à la mise en gavage est indispensable, car les mâles sont en général plus âgés que les femelles à ce moment. Les oisons de la première génération sont aussi mis au gavage en moyenne 2 jours plus tard que ceux de la seconde. Les effets des différences importantes d'âge moyen à la mise en gavage entre les 4 séries successives de gavage, surtout pour la $4^{\mathrm{e}}$ série, sont pris en compte aussi dans l'effet combiné série de gavage $\times$ gaveur.

En posant, pour les effets génétiques $g_{S E}$ et $g_{01}$ quels qu'ils soient, $g_{S E}+g_{01}=0$, l'on a donc $g_{S E-01}=2 g_{S E}$. L'on donnera comme résultats les $g_{S E}^{I}, g_{S E}^{M}, g_{S E}^{N}$ et $g_{S E}^{I s}$. Les hétérosis seront exprimés en écart entre les moyennes des croisés réciproques et des purs. Les fonctions estimables $h_{01 \times S E}^{I}$ et $h_{01 \times S E}^{M}$ s'appliquent aux moyennes des 2 sexes sous l'hypothèse de non différence entre sexes. La fonction estimable $g_{S E}^{I}$ estime en fait $g_{S E}^{I}+g_{S E}^{I s}$ si les effets directs de gènes liés au sexe existent. D'autre 


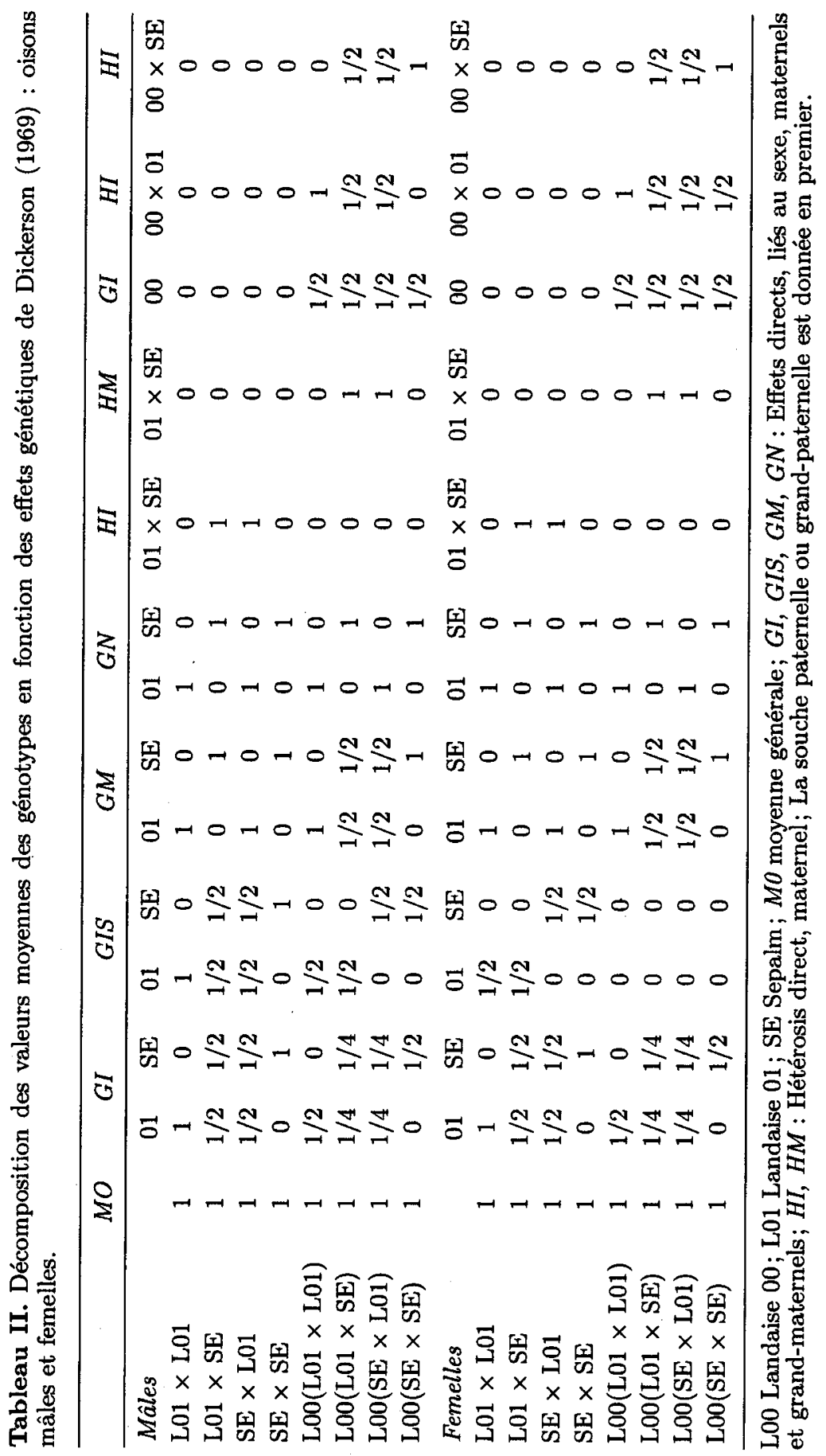


Tableau III. Expression des fonctions estimables utilisées pour estimer les paramètres génétiques du croisement.

\begin{tabular}{lcccccc}
\hline Génotypes & $H I$ & $H M$ & $G I$ & $G M$ & $G N$ & $G I S$ \\
\hline & $01 . \mathrm{SE}$ & $01 . \mathrm{SE}$ & $\mathrm{SE}$ & $\mathrm{SE}$ & $\mathrm{SE}$ & $\mathrm{SE}$ \\
$\mathrm{L} 01 \times \mathrm{L} 01$ & $-1 / 2$ & 0 & $-1 / 2$ & 0 & 0 & 0 \\
$\mathrm{~L} 01 \times \mathrm{SE}$ & $1 / 2$ & 0 & $-1 / 2$ & $1 / 2$ & 0 & $1^{\prime \prime}$ \\
$\mathrm{SE} \times \mathrm{L01}$ & $1 / 2$ & 0 & $1 / 2$ & $-1 / 2$ & 0 & $-1^{\prime \prime}$ \\
$\mathrm{SE} \times \mathrm{SE}$ & $-1 / 2$ & 0 & $1 / 2$ & 0 & 0 & 0 \\
$\mathrm{~L} 00(\mathrm{~L} 01 \times \mathrm{L} 01)$ & 0 & $-1 / 2$ & 0 & 0 & 0 & 0 \\
L00 $(\mathrm{L01} \times \mathrm{SE})$ & 0 & $1 / 2$ & 0 & $-1 / 2$ & $1 / 2^{+}$ & 0 \\
L00 $(\mathrm{SE} \times \mathrm{L} 01)$ & 0 & $1 / 2$ & 0 & $1 / 2$ & $-1 / 2^{+}$ & 0 \\
L00 $(\mathrm{SE} \times \mathrm{SE})$ & 0 & $-1 / 2$ & 0 & 0 & 0 & 0 \\
\hline
\end{tabular}

+ Chez les femelles seulement; " Différence mâles-femelles; L00, Landaise 00; L01, Landaise 01, SE, Sepalm; GI, GIS, GM, GN : Effets directs, liés au sexe, maternels et grand-maternels; $H I, H M$ : Hétérosis direct, maternel; La souche paternelle ou grandpaternelle est donnée en premier.

part, il existe des liens de parenté entre les individus mesurés. Ceux-ci n'étant pas pris en compte dans le modèle, les estimations ne sont plus les meilleures estimations linéaires sans biais, les erreurs standards des paramètres et les tests d'hypothèse ne sont plus strictement exacts, bien qu'il n'y ait pas eu de sélection des reproducteurs et que les effectifs de descendants mesurés de chaque reproducteur soient peu déséquilibrés. Komender et Hoeschele (1989) ont présenté une utilisation de la méthodologie du modèle mixte pour améliorer l'estimation des paramètres du croisement. Celle-ci prend en compte la matrice de coefficients de parenté entre les individus mesurés, mais elle suppose connues les variances génétiques. Elle suppose également, a priori, l'homogénéité entre les types génétiques purs et croisés des rapports des variances résiduelles aux variances génétiques. Ces hypothèses paraissent fortes pour l'application présente et compte tenu aussi de la théorie de la variabilité génétique conjointe en pur et en croisement $F_{1}$ de 2 populations (Brun et Rouvier, 1981).

\section{RÉSULTATS}

Les variances sont reconnues homogènes par un test de Bartlett pour le poids du foie, le taux de fonte lipidique, la fonte écart, les variables de gain de poids et la durée de gavage.

Les interactions entre le sexe et le type génétique ne sont jamais significatives; cela justifie d'estimer les paramètres sexes confondus. Les valeurs des coefficients de variation des caractères pondéraux des oisons sont comprises, quel que soit le sexe, entre $7,7 \%$ et $10,3 \%$. Celles des caractères de croissance en prégavage ou en gavage varient de $20,6 \%$ à $24,1 \%$. Le coefficient de variation du poids du foie est voisin de $25 \%$ et celui du taux de fonte de l'ordre de $42 \%$. La note de qualimètre est très 


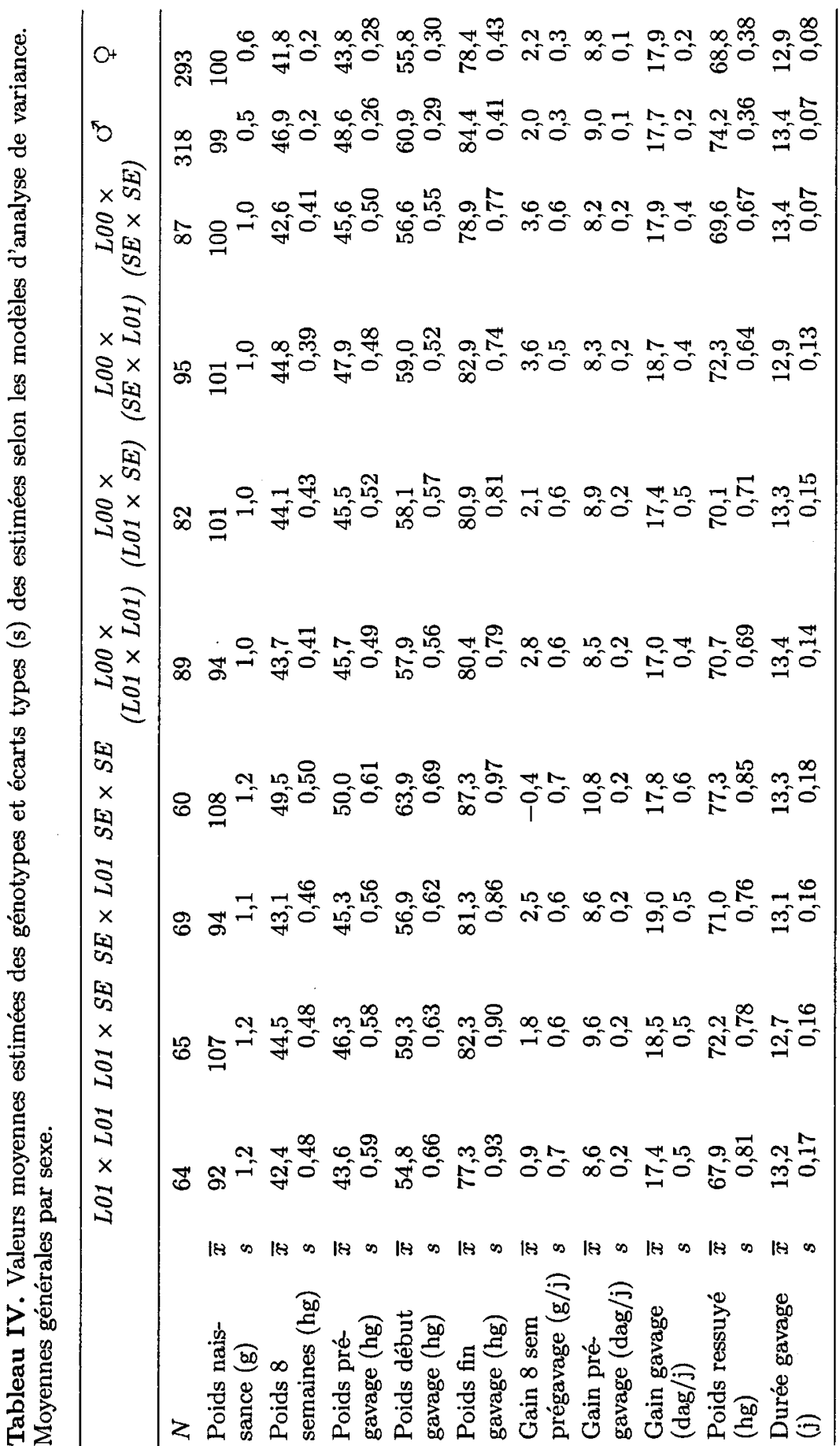




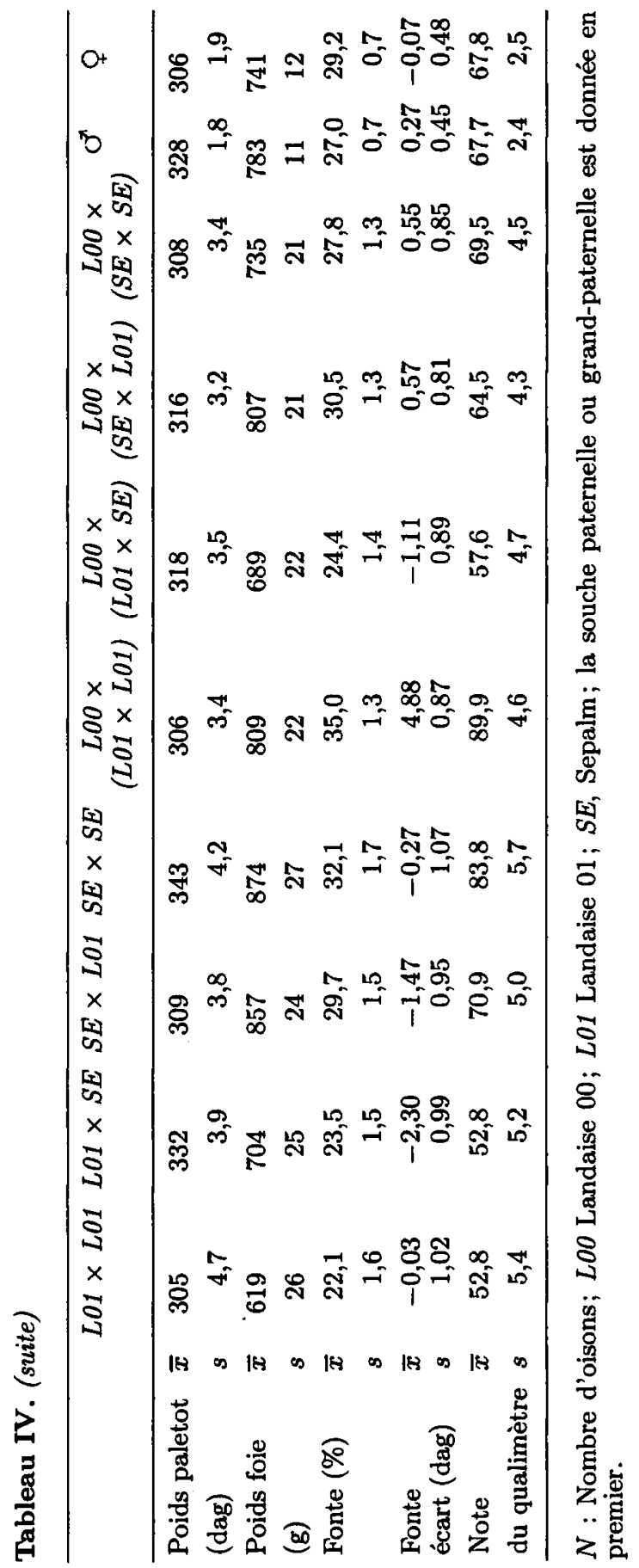


variable $(60 \%)$; la valeur du coefficient de variation de ce caractère est exacerbée par une réponse de l'appareil non linéaire pour les foies très peu fondants ou très fondants.

\section{Valeurs moyennes}

Les estimées des valeurs moyennes des génotypes, corrigées des effets sexe, série de gavage et gaveur et de l'effet de l'âge à la mise en gavage figurent au tableau IV. La souche pure Sepalm donne naissance aux oisons les plus lourds. Ceux-ci conservent leur avantage pondéral qui se traduit par un paletot et un foie plus lourds; sa croissance en période d'entretien ( 8 semaines-prégavage) est faible mais elle extériorise la meilleure croissance pondérale en période de prégavage. Les oisons métis des souches L01 et Sepalm ont des performances intermédiaires entre celles de leurs souches parentales, à l'exception d'une croissance plus élevée que celle de la moyenne des souches parentales en période d'entretien. Ils se distinguent, selon le sens du croisement, pour le poids du foie plus proche de celui de la souche paternelle, et le poids du paletot, plus proche de celui de la souche maternelle. Cette tendance se retrouve pour le poids du foie chez les métis trois souches. Les mâles sont plus lourds que les femelles et ce dès l'âge de 8 semaines. Ils donnent des foies gras et des paletots plus lourds. La différence mâles-femelles du poids du foie n'est pas significative dans le croisement SE $\times$ L01 (tableau V). Les foies gras des mâles fondent moins que ceux des femelles.

\section{Estimées des paramètres génétiques du croisement}

Le tableau VI indique les valeurs des estimées, de leurs écarts types et les significations statistiques des paramètres génétiques du croisement.

Un oison enfant d'un géniteur de la souche Sepalm, du fait des effets directs, est plus lourd dès sa naissance, gagne plus vite du poids, a un foie plus lourd dont la qualité technologique mesurée par le taux de fonte est amoindrie. Il atteint son optimum de gavage un peu plus tardivement. Par contre, à poids de foie constant, la fonte lipidique mesurée par la «fonte écart» n'est pas significativement augmentée. Les effets des gènes liés au sexe augmentent son poids à la naissance, le gain en cours de prégavage, le poids ressuyé, la durée du gavage, le poids du foie et les valeurs des indicateurs de qualité technologique. Pour tous les autres caractères on observe un antagonisme entre effet direct et effet lié au sexe. Cependant, si l'effet direct est le plus souvent statistiquement supérieur à 0 , l'effet lié au sexe n'est significatif que pour le gain en période de prégavage. Il est proche de la signification pour le taux de fonte lipidique.

Si l'oison est issu d'une mère de souche Sepalm, un antagonisme entre les effets maternels et grand-maternels modifie ses performances pondérales et le moment où il atteint son optimum de gavage. Le bilan positif de ces 2 effets renforce l'effet direct favorable de la souche Sepalm; un phénomène analogue régit les caractères de vitesse de croissance, à l'exclusion du croît en cours de prégavage, caractère pour lequel, comme pour le paletot, les effets maternels et grand-maternels, tous 2 positifs, renforcent l'effet direct favorable de la souche Sepalm. Un antagonisme fort entre les effets direct et grand-maternel en l'absence d'effet maternel significatif caractérise le poids du foie. Les effets maternels et grand-maternels de la souche 
Sepalm, de même signe, amoindrissent les effets directs positifs sur le taux de fonte; par contre, ils augmentent la fonte corrigée du poids du foie. Si l'oison est issu d'une grand-mère de la souche Sepalm, ses performances s'en trouvent le plus souvent diminuées par les effets grand-maternels; les exceptions sont : le gain en cours de prégavage, la durée du gavage, le poids du paletot et la fonte corrigée du poids du foie.

Tableau V. Moyennes de poids de foie gras \pm écarts types de la moyenne, estimées par les moindres carrés, chez les oisons mâles et femelles.

\begin{tabular}{lcc}
\hline & \multicolumn{2}{c}{ Poids du foie $(g)$} \\
Génotypes & Mâles & Femelles \\
\hline L01 $\times$ L01 & $652 \pm 33$ & $586 \pm 39$ \\
L01 $\times$ SE & $745 \pm 35$ & $664 \pm 35$ \\
SE $\times$ L01 & $861 \pm 33$ & $852 \pm 35$ \\
SE $\times$ SE & $909 \pm 36$ & $840 \pm 39$ \\
L00 $\times($ L01 $\times$ L01 $)$ & $822 \pm 32$ & $797 \pm 30$ \\
L00 $\times($ L01 $\times$ SE $)$ & $695 \pm 30$ & $684 \pm 33$ \\
L00 $\times($ SE $\times$ L01) & $819 \pm 29$ & $794 \pm 29$ \\
L00 $\times($ SE $\times$ SE $)$ & $759 \pm 31$ & $712 \pm 30$ \\
\hline
\end{tabular}

L00, Landaise 00; L01, Landaise 01 ; SE, Sepalm; la souche paternelle ou grand-paternelle est donnée en premier.

Les hétérosis directs du poids à l'âge de 8 semaines, de l'ensemble des gains de poids et de la durée de gavage sont statistiquement non nuls. Ils sont positifs pour les gains en période d'entretien ou en gavage et pour le poids du foie. Les hétérosis directs, positif sur le poids du foie, négatif sur la fonte lipidique sont non significatifs. Un oison métis des souches Sepalm et L01 a, en général, des performances de poids corporels inférieures à la moyenne des souches parentales; une qualité technologique du foie améliorée et une meilleure adaptation au régime d'alimentation avant le prégavage caractérisent les influences de l'hétérosis direct.

La plupart des hétérosis maternels sont significatifs et favorables. Ceux des gains en cours d'entretien ou de prégavage, du poids au début du prégavage et du poids du foie sont statistiquement nuls. À l'exclusion de la durée du gavage et des indicateurs de qualité technologique, il y a antagonisme entre les effets d'hétérosis direct et maternel. Un oison issu d'une mère métisse, par le jeu des hétérosis maternels, pèse plus lourd, a un foie un peu plus léger, de qualité technologie améliorée et atteint précocement son optimum de gavage.

\section{DISCUSSION}

À la différence du modèle habituellement utilisé pour estimer les paramètres génétiques du croisement, l'hypothèse d'effets génétiques directs de gènes liés au sexe a été introduite ici. Cela se justifie par leur importance a priori plus grande chez les oiseaux que chez les mammiferes. Depuis Dickerson (1969), Matheron et 


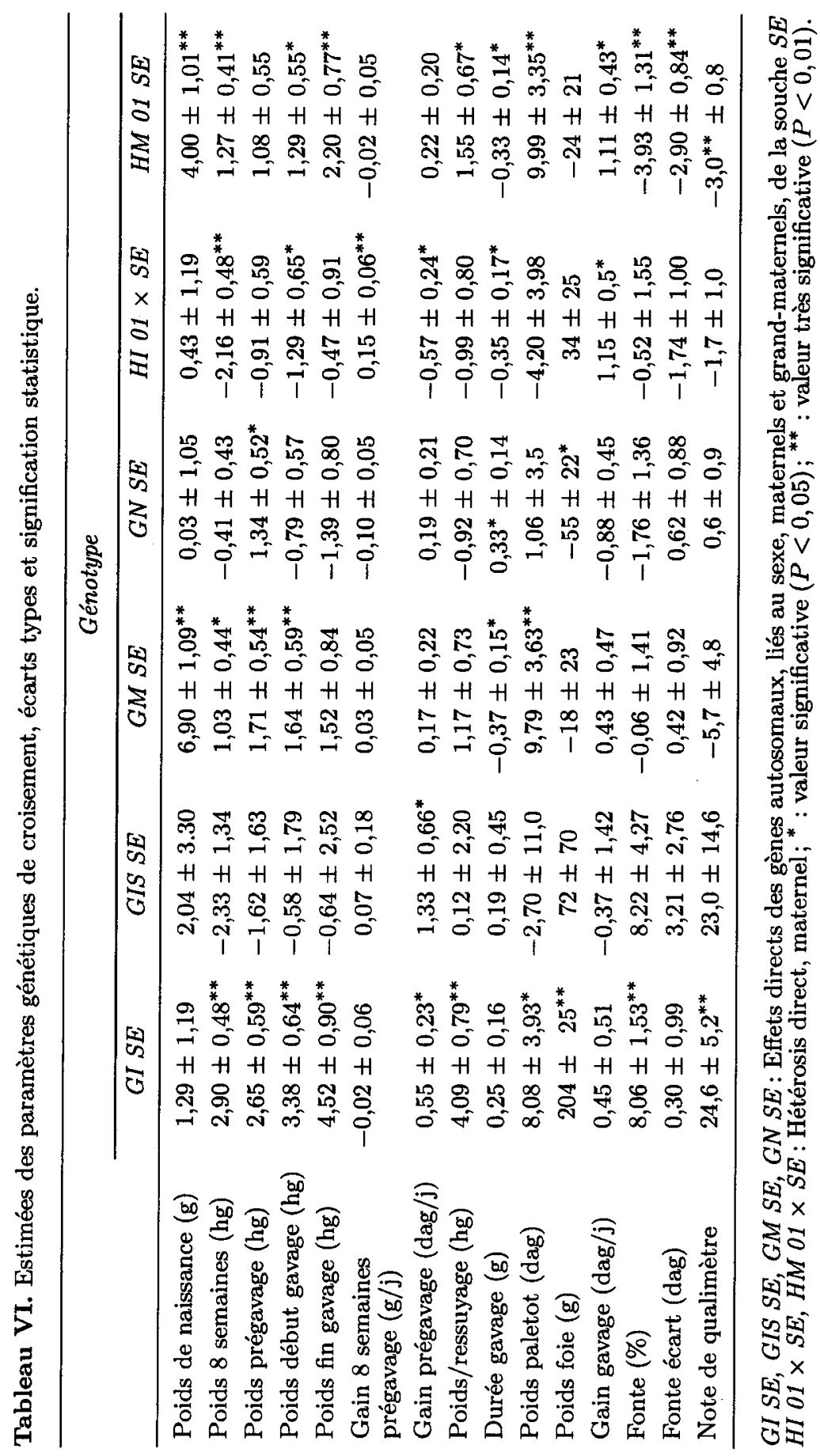


Mauleon (1979), Eisen et al (1983), les paramètres génétiques du croisement ont été largement estimés et discutés chez les mammiferes. Eisen et al ont donné une interprétation génétique des effets directs et maternels des gènes autosomaux, en l'absence d'épistasie, et précisé qu'ils peuvent contenir des effets de dominance si elle existe. Les effets maternels et grand-maternels sont aussi appelés effets indirects des gènes. La discussion va privilégier l'interprétation des effets génétiques mis en évidence pour une meilleure connaissance du déterminisme génétique des caractères de production de foie gras dans ces souches, et sur le plan des applications pour la recherche de leur système d'utilisation optimale.

\section{Effets non génétiques et effets du sexe}

L'effet du sexe confirme le dimorphisme sexuel en poids en faveur des mâles, déjà mis en évidence (Rouvier et al, 1982a) dans les souches L00 et L01. Cependant, la différence entre sexes de gain en gavage n'est pas confirmée en moyenne sur cet échantillonnage plus vaste de types génétiques. L'effet du sexe sur le poids du foie gras varie suivant les souches et les croisements, ce qui s'expliquera par l'existence d'effets de gènes liés au chromosome sexuel, ainsi que par les effets génétiques maternels et grand-maternels.

La relation moyenne positive entre taux de fonte lipidique et poids du foie gras est inversée entre sexes puisque, en moyenne, les foies gras des mâles sont plus lourds et moins fondants que ceux des femelles. Les raisons devront en être approfondies.

\section{Valeurs moyennes}

Il faut d'abord noter une réduction de la durée du gavage par rapport aux résultats publiés sur les 2 souches L00 et L01: 13 j contre 18 j en 1982. Cela peut s'expliquer par la sélection réalisée pour la stéatose hépatique, et par une modification de la technique d'élevage, de prégavage qui conduit à un gavage plus intensif. En effet, le gain de poids journalier en gavage est accru, le gain journalier moyen en prégavage est élevé, le gain moyen journalier entre l'âge 8 semaines et la mise en prégavage est devenu voisin de 0 . La technique d'élevage et d'alimentation maintenant utilisée essaie d'obtenir une croissance optimale jusqu'à l'âge de 8 semaines, puis de maintenir le poids des oisons jusqu'à la mise en prégavage. La quantité d'aliment ingéré par repas en gavage est accrue.

Le poids de foie gras élevé dans la souche Sepalm est lié à son mode de constitution, à son poids corporel et au progrès réalisé dans cette souche grâce à la sélection. Le poids du foie gras dans la souche L01 n'a pratiquement pas varié par rapport aux résultats de Rouvier et al (1982a) : 619 contre $610 \mathrm{~g}$.

\section{Effets génétiques directs, maternels, grand-maternels}

Les jars Sepalm utilisés en pur ou en croisement avec l'oie L01 sont améliorateurs pour le poids du foie gras. Un effet génétique direct existe sur le taux de fonte, mais pas sur la fonte à poids de foie constant («fonte écart»). Il est intéressant de discuter les mécanismes physiologiques possibles. Leclercq et al (1968) étudient l'évolution de constituants biochimiques du foie d'oies de race Masseube au cours du gavage et concluent que l'organe est le siège à la fois d'une hyperplasie et d'une hypertrophie. 
Blum et Leclercq (1973) précisent qu'il n'y a pas hyperplasie, mais seulement hypertrophie du tissu hépatique. La stéatose hépatique est une accumulation de graisses, principalement des triglycérides, dans les cellules hépatiques. Nir et Nitsan (1976), à partir d'oies gavées de plusieurs types génétiques, indiquent l'existence d'une variabilité dans les pourcentages de graisses et de protéines par rapport au poids du foie gras. Le taux de fonte lipidique mesurée par ces auteurs est corrélé positivement avec le pourcentage de lipides totaux, négativement avec ceux de protéines et de phosphore total, Blum et al (1990) obtiennent des résultats comparables.

La fonte augmente lorsque la surcharge graisseuse devient trop importante par rapport à la «masse active» du foie constituée de protéines et de phospholipides. Contrairement au cas des mammifêres, le foie est le principal site de la lipogénèse chez les oiseaux (Saadoun et Leclercq, 1983; cités par Hermier et al, 1988). Les triglycérides, synthétisés in situ à partir des hydrates de carbone, sont relâchés dans la circulation sanguine sous forme de VLDL. Une déficience relative dans la production de VLDL pourrait conduire à une stéatose hépatique plus rapide et plus importante (Hermier et al, 1988, 1991).

On peut donc imaginer plusieurs voies d'actions non antagonistes des gènes sur le poids et la qualité technologique des foies gras. La sélection des souches sur le poids du foie gras, caractère de forte héritabilité (résultats non publiés), se traduirait aussi par une augmentation de la masse active. Lorsque la surcharge graisseuse devient proportionnellement trop grande, les foies deviennent plus fondants. Ce mécanisme pourrait aussi expliquer la différence entre mâles et femelles. Seule une étude ultérieure des effets génétiques régissant la croissance du tissu hépatique depuis la naissance et la composition biochimique des foies permettrait d'élucider cette question.

L'effet génétique de la souche Sepalm sur le poids du paletot est lié à celui sur la croissance squelettique et musculaire dans cette souche de plus grande taille que la souche L01. Cette différence du format s'accompagne-t-elle d'une cellularité supérieure des foies? Nous obtiendrions alors des foies plus lourds à surcharge lipidique égale.

La mère L01 des oisons de père Sepalm améliore le poids du foie gras, surtout chez les femelles, sans détérioration de la qualité technologique mesurée par la «fonte écart». Des effets de gènes liés au sexe et une complémentarité entre effets génétiques directs de la souche Sepalm d'une part, maternels et grand-maternels de la L01 d'autre part, sur ces 2 caractères, sont mis en évidence. Il s'ensuit que les oisons issus du croisement entre le jars Sepalm et l'oie L01 produisent des foies gras lourds sans dimorphisme en poids entre les 2 sexes, donc homogènes et de qualité technologique améliorée.

La mère de l'oison à un effet important sur le poids du paletot. Cela explique le plus faible poids de paletot dans le croisement entre le jars Sepalm et l'oie L01.

\section{Hétérosis direct et maternel}

L'hétérosis direct sur la croissance entre l'âge de 8 semaines et la mise en prégavage est très élevé. Les oisons croisés $F_{1}$ sont plus aptes à l'utilisation d'une quantité d'aliment réduite pendant la période d'entretien. En l'absence de compétition pour 
accéder à l'aliment, il est possible que les gènes agissent par surdominance sur l'utilisation digestive dans le cas d'une alimentation restreinte. De plus, les oisons $F_{1}$ sont avantagés par rapport aux oisons Sepalm parce qu'ils sont plus légers; leurs besoins d'entretien sont plus faibles. Par contre, les hétérosis directs sont négatifs pour le poids vif à 8 semaines, le gain pendant la période de prégavage et le poids à la mise en gavage. En période d'alimentation à volonté, il est possible que les oisons $F_{1}$ aient une meilleure homéostasie et ne surconsomment pas spontanément. De façon générale, c'est le sexe hétérogamétique qui bénéficie le plus, ici, de l'hétérosis maternel. Les hétérosis maternels sur le poids à 8 semaines peuvent être interprétés comme une conséquence de l'hétérosis maternel sur le poids à la naissance. Les œufs des oies $F_{1}$ assureraient un meilleur développement embryonnaire des oisons par leur valeur nutritive pour l'embryon, puisque l'hétérosis direct sur le poids à la naissance est nul.

Le poids du foie gras est soumis uniquement aux effets directs et indirects maternels et grand-maternels des gènes. Par contre, le croisement a un effet favorable pour diminuer la fonte lipidique du fait d'effets d'hétérosis direct et maternel négatifs. Cela pourrait être lié à une plus grande solidité des membranes cellulaires hépatiques chez ces métis.

Les estimées des effets génétiques sur la note de qualimètre vont dans le même sens que celles des effets génétiques sur le taux de fonte lipidique. Cela indique la valeur prédictive en moyenne sur le plan qualitatif de cette mesure.

\section{CONCLUSION}

Les caractères de croissance pondérale sont soumis aux actions conjointes des effets directs et indirects des gènes et d'hétérosis.

Des différences existent entre types génétiques, non seulement pour le poids du foie gras, mais aussi pour la fonte lipidique à la stérilisation. Le poids du foie gras dépend des effets génétiques directs et indirects, et peu de leurs interactions, alors que la fonte lipidique est, de plus, régie par un hétérosis maternel négatif. L'effet direct des gènes liés au sexe est proche de la signification pour le taux de fonte lipidique. Les effets directs et indirects des gènes conditonneraient la croissance de la masse active du foie. Chez les métis, il pourrait y avoir une plus grande solidité des membranes des hépatocytes. Ces interprétations seraient à vérifier sur les plans biochimique et expérimental. Il serait également intéressant de déterminer par quels mécanismes les foies gras des oisons mâles, plus lourds en moyenne que ceux des femelles, ont, en moyenne, une fonte lipidique à la stérilisation plus faible. Les effets génétiques directs des gènes autosomaux et liés au sexe et les effets génétiques indirects agissent par complémentarité chez les métis $\mathrm{SE} \times \mathrm{L} 01$, conduisant à des foies gras plus lourds, sans le dimorphisme sexuel en poids observé dans d'autres cas, et qui ne sont pas relativement plus fondants que des foies gras plus légers. La sélection et le croisement peuvent donc permettre d'améliorer simultanément le poids et la qualité technologique des foies gras d'oie. 


\section{REMERCIEMENTS}

Nous remercions la Sica Sepalm, Centre de Sélection des Palmipèdes gras, Souprosse, F 40250 , qui a fourni les reproducteurs de la souche Sepalm pour cette expérimentation et a suggéré cette étude, ainsi que les lecteurs du premier manuscrit pour leurs critiques constructives qui ont permis de l'améliorer.

\section{RÉFÉRENCES}

Bartel M (1984) Contribution à la recherche de mesures objectives de la qualité des foies gras d'oies. Thèse Doctorat vétérinaire, Université Paul Sabatier, Toulouse, $83 \mathrm{p}$

Blum JC, Leclercq B (1973) Nouvelles précisions sur les modifications biochimiques et histologiques du foie provoqués par le gavage. In : Volume II degli Atti delle Giornate Avicole Internazionali Varese, 31 Maggio-4 Giugno 1973, 193-207

Blum JC, Labie C, Raynaud P (1990) Influence du poids et de la composition chimique du foie gras d'oie sur la fonte mesurée après stérilisation à $104^{\circ} \mathrm{C}$. Sci Alim 10, 537-548

Brun JM, Rouvier R (1981) Eléments de génétique quantitative d'une population $F_{1}$ issue du croisement de deux populations. In : Variabilité génétique conjointe en pur et en croisement $F_{1}$ de deux populations. Applications à la sélection animale. Toulouse 13-14 Octobre 1981, INRA Publ, 1982, Les Colloques de l'INRA, n¹0, 55-64

Brun JM, Rouvier R (1984) Effets génétiques sur les caractères des portées issues de trois souches de lapins utilisées en croisement. Génét Sél Evol 16, 367-384

Brun JM, Rouvier R (1988) Paramètres génétiques des caractères de la portée et du poids de la mère dans le croisement de deux souches de lapins sélectionnées. Génét Sél Evol 20, 367-378

Dickerson GE (1969) Experimental approaches in utilizing breed resources. Anim Breed Abstr 37, 191-202

Eisen EJ, Horstgen Schark G, Saxton AM, Bandy TR (1983) Genetic interpretation and analysis of diallel cross with animals. Theor Appl Genet 65, 17-23

Hermier D, Forgez P, Laplaud PM, Chapman MJ (1988) Density distribution and physicochemical properties of plasma lipoproteins and apolipoproteins in the goose, Anser anser, a potential model of liver steastosis. J Lipid Res 29, 893-907

Hermier D, Saadoun A, Salichon MR, Sellier N, Rousselot-Paillet D, Chapman MJ (1991) Plasma lipoproteins and liver lipids in two breeds of geese with different susceptibility to hepatic steatosis : changes induced by development and forcefeeding. Lipids 26, 331-339

Komender P, Hoeschele I (1989) Use of mixed model methodology to improve estimation of crossbreeding parameters. Livest Prod Sci 21, 101-113

Leclercq B, Durand G, Delpech P, Blum JC (1968) Note préliminaire sur l'évolution des constituants biochimiques du foie au cours du gavage de l'oie. Ann Biol Anim Biochim Biophys 8, 549-556

Matheron G, Mauleon P (1979) Mise en évidence de l'action conjointe des effets directs, maternels et grand-maternels sur la taille de portée. Bull Tech Dép Génét Anim INRA Fr 29-30, 232-274 
Menissier F, Bibé B, Perreau B, Vissac B (1988) Comparaison des races bovines Chaloraise, Limousine et Maine-Anjou en race pure et en intercroisement. 1. Objectif et dispositif expérimental. Génét Sél Evol 20, 321-342

Nir I, Nitsan Z (1976) Goose fatty liver composition as related to the degree of steatosis, nutritional and technological treatments, and a simplified method for quality estimation. Ann Zootech 25, 461-470

Rouvier R, Vrillon A, Rousselot-Pailley D, Larrue P (1982a) Étude de quelques facteurs de variation du poids à deux mois et des performances de prégavage et de gavage d'oies Landaises en station. Ann Génét Sél Anim 14, 381-398

Rouvier R, Vrillon A, Rousselot-Pailley D, Larrue P (1982b) Efficacité théorique d'un indice de sélection sur collatéraux pour le foie gras de l'oie Landaise. Ann Génét Sél Anim 14, 245-252

Salladarre P (1978) Objectivation des qualités technologiques des foies gras par mesure d'impédance bioélectrique. Thèse Docteur-Ingénieur, Univ Claude Bernard, Lyon, $\mathbf{n}^{\circ} 296$ 